\title{
La formación integral del estudiantado de ingeniería a través de la educación continua
}

\author{
Comprehensive Training of Engineering Students \\ through Continuing Education
}

\author{
Miguel Reynoso Flores ${ }^{1}$ \\ Universidad Autónoma de Nuevo León \\ Nuevo León, México \\ miguel.reynosof@uanl.mx \\ Jaime Arturo Castillo Elizondo ${ }^{2}$ \\ Universidad Autónoma de Nuevo León \\ Nuevo León, México \\ Jaime.castilloe@uanl.mx \\ María Isabel Dimas Rangel ${ }^{3}$ \\ Universidad Autónoma de Nuevo León \\ Nuevo León, México \\ Isabel.dimasr@gmail.com
}

Recibido 4 de octubre de 2013 • Corregido 29 de octubre de 2013 • Aceptado 6 de noviembre de 2013

1 Ingeniero Administrador de Sistemas. Coordinador de Educación Continua. Doctor en Educación (México, 2013). Master en Ciencias de la Administración. Ha publicado artículos en revistas referenciadas, es autor de capítulos de libros y ha presentado varios trabajos en congresos educativos.

2 Ingeniero Administrador de Sistemas en la FIME de la UANL. Maestría en Administración con especialidad en Recursos Humanos. Profesor de tiempo completo con Perfil PROMEP otorgado por la Secretaría de Educación Publica de México. Experiencia en el sector empresarial y educativo para las áreas de sistemas, administración, gestión y vinculación. Actualmente subdirector de Vinculación y Relaciones de la Facultad de Ingeniería Mecánica y Eléctrica. Ha presentado y publicado diversos trabajos relacionados con el tema de la vinculación y la gestión en revistas de prestigio y congresos internacionales.

3 Maestra en Ciencias de la Administración, de la Universidad Autónoma de Nuevo León (UANL). Jefa de Recursos Humanos de la Facultad de Ingeniería Mecánica y Eléctrica (FIME). Profesora titular. Miembro de la Academia de Manufactura y Calidad. Experiencia como investigadora en el área de gestión de recursos humanos y sistemas de calidad. Posee artículos publicados en las áreas de gestión de recursos humanos, gestión de procesos con base en sistemas de calidad, en revistas de prestigio internacional. 
Resumen. El presente artículo aborda un tema prioritario para la formación del estudiantado en general y en particular para los futuros ingenieros e ingenieras. A pesar de que en los últimos años se ha abordado con frecuencia esta temática; aún son insuficientes las propuestas en el caso del estudiante de ingeniería. El objetivo de este artículo es fundamentar teórica y empíricamente las potencialidades que posee la educación continua como una de las áreas fundamentales que poseen las facultades de ingeniería para la formación integral de sus estudiantes. Se muestran resultados preliminares de un proyecto de investigación solicitado por la Facultad de Ingeniería Mecánica y Eléctrica (FIME) de la Universidad Autónoma de Nuevo León, México, a partir de la necesidad de perfeccionar el proceso formativo de los estudiantes con un enfoque integral. Se expone la justificación del estudio y se presentan algunos de los resultados obtenidos en la fase exploratoria.

Palabras claves. Formación integral del ingeniero, educación continua, estudiante de ingeniería

Abstract. This paper addresses a priority for student training in general and particularly for future engineers. Although this issue has been frequently addressed in recent years, proposals are still insufficient for engineering students. This paper is aimed at theoretically and empirically demonstrating the potential of continuing education as one of the key areas that engineering schools have for the comprehensive training of students. Preliminary results of a research project commissioned by the School of Mechanical and Electrical Engineering (Facultad de Ingeniería Mecánica y Electrica-FIME) of Universidad Autónoma de Nuevo León, Mexico, are presented to respond to the need to improve the learning process of students with a comprehensive approach. The research justification and some of the results obtained in the exploratory phase are also described.

Keywords. Comprehensive training of engineers, continuing education, engineering student

Es conocido que el creciente desarrollo de la sociedad se caracteriza hoy día por la intensificación de los problemas económicos, políticos y sociales que, unidos a fenómenos naturales, ejercen sus efectos en diferentes ecosistemas del planeta, esta situación ha influido negativamente en la calidad de vida no solo del ser humano, sino también de las diferentes especies de plantas y animales.

El desarrollo vertiginoso que la ciencia y la técnica han tenido en los últimos años exige a las instituciones de educación superior la adopción de nuevas concepciones y enfoques en el proceso de formación de los profesionales, de tal manera que sean capaces de elevar los niveles científicotecnológicos. Sin embargo, no ocupa el mismo interés en las políticas educativas la necesidad de formar profesionales, ante todo con un compromiso ético hacia el desarrollo humano.

Muy relacionado con lo anterior se encuentra la necesidad del perfeccionamiento de la formación de docentes, de modo tal que puedan ejercer una influencia positiva y significativa en sus estudiantes. Como destacan Soto, Dimas y Torres (2012):

Se aprecia que el profesorado universitario en México hoy día es un colectivo amplio y complejo, cuyos múltiples talentos deben estimularse de forma tal que su formación constituye un área de oportunidad para la confección de una estrategia que contribuya al fortalecimiento personal y profesional del docente. (p. 256) 
Con estas condiciones, la universidad como la institución social responsabilizada con el desarrollo al más alto nivel de los recursos humanos de cualquier país, es la que prepara a los profesionales para enfrentarse a los crecientes retos en las esferas científico- técnico y cultural. De ahí el ininterrumpido proceso de perfeccionamiento de la educación superior, el cual se expresa de diferentes formas y en diversas áreas. Por esta razón, la formación de docentes y estudiantes continúa en el centro de atención de las investigaciones sociales y pedagógicas.

Por lo antes expresado apunta Álvarez (2013):

Por más vueltas que se dé [sic] el centro de Pedagogía no puede ser otro que los dos sujetos reconocidos desde la antigüedad como una unidad indisoluble: maestros (as) y estudiantes. Con el uso de tecnologías o sin ellas la tipología del docente y la interacción con sus estudiantes continuará siendo un problema tan importante [y actual] como la relación médico-paciente. (p. 18)

En el contexto universitario, la educación posee un significado especial: para educar a "ser más para ser mejor", y en "madurez humana", apuntan De la Herrán y Muñoz (2002, p. 52). En este sentido, se acentúa la necesidad de potenciar el desarrollo humano en la formación con base en sólidos fundamentos éticos, pues "así como la tecnociencia contemporánea penetra todas las actividades del ser humano y de la sociedad, así la ética centrada en la persona humana 'permea' todos los desarrollos de la ciencia y de la tecnología" (Cornejo et al., 2013, p. 13).

A estas exigencias no escapa el estudiantado de ingeniería, el cual, una vez graduado, ha de ejercer una influencia positiva en la solución de problemas científico-tecnológicos y sociales que influyan de manera decisiva en el desarrollo de la sociedad. Lo anterior ha postula que:

La formación de ingenieros capacitados para enfrentar los retos del siglo XXI, constituye un desafío para las universidades hoy día. El desarrollo científico y tecnológico de esta era, tales como la nanotecnología, redes de información, la ingeniería genética, superestructuras, exigen profesionales altamente calificados y capacitados para su manejo. (Méndez, 2007, p. 1)

Dadas las actuales exigencias sociales, las instituciones universitarias en México tienen la misión de potenciar un estudiantado crítico, analítico, innovador y con un alto nivel de desarrollo de los valores humanos. El interés se centra desde la actualización de un volumen de información considerable, hasta propiciar la autogestión del aprendizaje que tribute a su formación integral, a partir de las acciones que ejecuta el personal docente en su calidad orientadora y mediadora, en aras de la concientización de la necesidad del estudiantado de crecer psicológicamente, de compartir con sus semejantes en un contexto donde el "saber convivir" resulta imprescindible. 
URL: http://www.una.ac.cr/educare

CORREO: educare@una.cr

También es un imperativo desarrollar la reflexión y el análisis crítico como vías para aprender de manera más significativa y comprender mejor la realidad circundante $y$, en consecuencia, mejorarla de modo permanente.

Las citadas aspiraciones demandan de propuestas que contribuyan a su concreción, así como a la eliminación de aquellas prácticas educativas nocivas que aún persisten. En aras de explorar algunos de los citados aspectos fueron aplicadas encuestas en el curso (2011-2012) en la FIME, mediante las cuales se obtuvieron resultados donde afloran problemas evidentes que requieren ser solucionados a la mayor brevedad. A pesar de la aplicación de un nuevo modelo que enfatiza en la "formación por competencias", se observan una serie de dificultades que afectan el proceso formativo integral del estudiante, tales como:

a) Estilos comunicativos autocráticos en el actuar de ciertos docentes.

b) Labor educativa que no satisface completamente las expectativas institucionales, ni las auténticas demandas de la formación integral profesional.

c) Frecuente preocupación fundamental en el proceso formativo, el cumplimiento del programa de las asignaturas.

d) Carácter informativo de las clases, centradas fundamentalmente en el personal docente y el contenido del programa.

e) Insuficientes estrategias formativas para la inclusión estudiantil en actividades que contribuyan a su formación integral.

Lo expuesto justifica la necesidad de continuar investigando en esta problemática. Por ello, el presente artículo realiza una justificación de su estudio y expone los resultados preliminares de un proyecto de investigación sobre la formación integral del estudiantado de ingeniería, resultados que muestran los datos fundamentales de la etapa exploratoria.

La base metodológica que sustenta la investigación es el método dialéctico como método general, el cual permite penetrar en las relaciones y contradicciones que ocurren en el proceso de formación del estudiantado de ingeniería, tales como: la relación entre lo universal, lo particular y lo singular, las relaciones causa-efecto; así como en la esencia transformadora que posee el estudiantado en su vínculo directo con los demás y el medio que le rodea. Se conjugan métodos teóricos como el histórico-lógico, análisis síntesis con la aplicación de encuestas a estudiantes y docentes de la Facultad.

La muestra estuvo integrada por estudiantes de las 10 carreras de la Facultad tomados al azar, correspondiente al $10 \%$ del total. Asimismo, se tomó una muestra intencional de profesores de las diferentes carreras, en total 60 docentes. 


\section{La formación en ingenierías}

El campo profesional de la ingeniería es sumamente amplio, por lo que su importancia en el desarrollo social y científico-tecnológico es esencial; por esta razón, "la ingeniería se encuentra ante un abanico de grandes retos para desarrollar nuevos avances que disminuyan los problemas que enfrenta la sociedad en factores como el ambiente, el suministro de vivienda, el agua y el cuidado de la salud para una población que crece rápidamente" (León y Ramírez, 2010, p. 5).

De acuerdo con lo anterior, la enseñanza de las diferentes especialidades de ingeniería no puede estar ajena a los retos que requiere el contexto de la sociedad actual. Fuera de los muros universitarios existe un mercado de trabajo que demanda, cada vez más, no solo una persona egresada hábil y capaz, sino también "competente". Si esto no se logra, posiblemente las economías de los países en desarrollo sucumbirán ante el poderío hegemónico y globalizador de los países más desarrollados.

Para el logro de los propósitos apuntados, se concuerda con Álvarez (2002) acerca de que "las instituciones de educación superior deben formar a los estudiantes para que se conviertan en ciudadanos bien informados y profundamente motivados, provistos de un sentido crítico y capaz de analizar los problemas, buscar soluciones para los que se planteen a la sociedad, aplicar éstas y asumir responsabilidades sociales" (p. 4).

Sin embargo, tal y como se expresa en el documento La educación superior para el siglo XXI. Líneas estratégicas de desarrollo (Asociación Nacional de Universidades e Instituciones de Educación Superior de México [ANUIES], 2000):

Los estudiantes de la educación superior no han sido en México objeto prioritario de investigación. Pese a su importancia, a este actor se le conoce mal y poco (antecedentes, situación social, económica y cultural, trayectorias, etc.). Quienes se han acercado a su conocimiento lo han hecho en los momentos de alta politización o de conflictos, pero se han perdido de vista las enormes transformaciones ocurridas en ellos en los últimos años". (p. 108)

Se complementa la idea anterior cuando se expresa en el citado documento que:

Son incipientes los programas especiales para estudiantes de alto rendimiento académico y no se ha atendido suficientemente la formación integral del estudiante, sobre todo en lo que se refiere a la formación en los valores deseables para tener los ciudadanos responsables que requiere el desarrollo de la nación, así como en los principios éticos de cada una de las profesiones. (ANUIES, 2000, p. 110) 
URL: http://www.una.ac.cr/educare

CORREO: educare@una.cr

La XXVII Conferencia Nacional de Ingeniería, celebrada en la ciudad de Toluca, México (ANFEl, 2000), se dedicó al tratamiento del tema "La formación humanística del ingeniero". En sus conclusiones destaca que entre las deficiencias que deben subsanarse para lograr esa formación humanística y, como consecuencia integral, está la falta de interacción humana por parte de los estudiantes, dificultad para expresarse oralmente y por escrito, así como elocuencia para defender y sustentar sus ideas; por otra parte, se considera que en el país los jóvenes no encuentran modelos para seguir, por lo tanto, la manifestación de valores no siempre está presente.

En el marco de la citada conferencia se puntualizaron requisitos muy importantes para la formación integral del estudiantado de ingeniería. Se propuso que egresados y egresadas de estas carreras deberán ser capaces de:

a) Vivir en la incertidumbre del cambio acelerado de la ciencia y tecnología

b) Preservar el ambiente

c) Practicar el ejercicio de la indagación científica

d) Atender las necesidades sociales

e) Fomentar la solidaridad e igualdad

Los requisitos antes expuestos se relacionan con las competencias genéricas que deben tener los estudiantes de ingeniería una vez egresados. La comprensión de la importancia de esta es algo sustancial para el logro de una formación que trascienda los límites de una determinada preparación profesional.

El Consejo Federal de Decanos de Ingeniería (CONFEDI) (2006, p. 12) precisó las principales competencias para ingeniería, entre las que destacan:

Competencias tecnológicas:

1. ... Identificar, formular y resolver problemas...

2. ...Diseñar y controlar proyectos...

3. ...Gestionar, planificar, ejecutar y controlar proyectos de ingeniería...

4. ... Utilizar de manera efectiva las técnicas y herramientas...

5. [Generar] desarrollos tenológicos y/o [sic] innovaciones tecnológicas 
Competencias sociales, políticas y actitudinales:

6. Trabajar en equipo

7. Comunicarse de manera efectiva

8. Actuar en forma ética y responsable

9. Aprender de manera continua

10. Actuar con espíritu emprendedor

En las facultades de ingeniería en México existen múltiples vías que permiten contribuir al desarrollo de las citadas competencias y, como consecuencia, a la formación integral de sus estudiantes. Una de ellas es la educación continua que, en el caso de la FIME, constituye una de sus áreas importantes y posee el rango de coordinación.

\section{La educación continua, sentido y significado}

La educación continua fue creada con el fin de integrar un espectro de actividades y programas de aprendizaje teórico y práctico que se aplican después de la formación obligatoria o reglamentada (ya sea de educación secundaria, del bachillerato, la formación profesional o de la universidad) y que puede extenderse durante toda la vida. También puede entenderse como educación para adultos, aunque supera este concepto ya que incluye jóvenes, profesionales, adultos y ancianos (universidad de la experiencia) que, con titulaciones oficiales o sin ellas, profundizan en su educación y formación. Cuando los programas de convalidación o correspondencia existen, pueden alcanzar reconocimiento oficial con la titulación pertinente.

\section{Breve historia de la educación continua en la FIME}

La historia de la educación continua como coordinación está ligada a la historia de la Facultad, porque ha participado activamente en la consecución de sus objetivos principales. Desde sus orígenes ha ofrecido formación técnica, diplomados, talleres y cursos que promueven el crecimiento profesional, con un programa actualizado para la capacitación técnica de los individuos en la sociedad y en el mundo laboral.

Esta ha sido una de las principales fortalezas, por una parte, la adecuación al entorno económico social y por la otra, la preparación de personas con el nombramiento de técnicos y especialistas en diversas áreas.

Asimismo, a través del tiempo, dicha coordinación ha tenido un creciente desarrollo tanto en estudiantes e instructores como en instalaciones y programas técnicos y de desarrollo 
profesional. Entre sus objetivos está el establecer un programa de capacitación que responda a las necesidades de las empresas del ramo mecánico, eléctrico y de software, con finalidad de satisfacer las exigencias que estas demandan.

En el 2008, el el Departamento de Educación Continua pasó a ser una coordinación, tomando en cuenta que la proyección de los servicios a la industria y al déficit de técnicos en Nuevo León exigía un trabajo más abarcador. Este estado se caracteriza por ser muy competitivo, debido principalmente, a la rapidez del cambio e innovación de la tecnología, a la diversidad de las estructuras empresariales y de la organización del trabajo, así como a la globalización de los mercados, en un alto entorno empresarial y nivel de industrialización.

En la actualidad, el incremento notable de la oferta de programas de actualización profesional, laboral y desarrollo personal, tanto en las universidades como en los centros de enseñanza no universitaria, ha indicado el enorme interés existente para estas actividades que fomentan el conocimiento y las actitudes necesarias para la superación. Este hecho ha provocado que gran parte de la oferta de los programas sea brindada por universidades o instituciones no universitarias. Este hecho ocurre porque con frecuencia las facultades de ingeniería demoran en dar respuesta a las necesidades de este tipo de programas, de manera que, en la actualidad, es insuficiente el cumplimiento de las exigencias planteadas por los centros empleadores.

La educación continua constituye una formación permanente identificada, frecuentemente, con este último término porque se relaciona con una vía para completar la formación inicial del individuo. El objetivo de este tipo de educación se justifica por el hecho de que las competencias del estudiantado -que integran conocimientos, habilidades, actitudes, destrezas y juicios necesarios para dispensar servicios profesionales pertinentes, seguros y oportunos a una población dada- exigen de constante actualización y perfeccionamiento.

Aunque lo apuntado no debería ser el único fin de la educación continua, esta concepción se mantiene vigente debido a que la educación inicial, independientemente de su duración, no garantiza un ejercicio profesional idóneo, sino que capacita solo para comenzar una carrera o empleo y, en ocasiones, no aporta los cimientos para continuar la educación durante toda la vida profesional. Por esta razón, indica Mejía (1986) que sin educación continua la competencia decrece progresivamente como consecuencia de una dinámica influida por los siguientes factores.

Según la ANUIES (2000), la educación continua se considera la actividad académica de extensión universitaria que se organiza y ubica fuera del sistema formal educativo; tiene como propósito actualizar conocimientos, y adquirir nuevas destrezas y habilidades que permitan una mejor adaptación al cambio y un desempeño eficiente en el entorno laboral.

La educación continua se identifica también como formación continua y, generalmente, se valora como una vía para la actualización profesional con un marcado sentido técnico e instructivo tal y como se observa cuando se expone que: 
La formación continua (FC) es aquella formación que recibe una persona después de haber finalizado su formación inicial en una profesión, con el fin de ampliar o perfeccionar sus competencias profesionales. Por lo tanto, es la formación dirigida a los profesionales en activo que pretenden mejorar sus conocimientos, habilidades y actitudes para el desarrollo de su profesión. Este tipo de formación se da a lo largo de la vida laboral de la persona, por lo que se vincula con el concepto de educación permanente y de ahí su adjetivación como "continua". (Pineda y Sarramona, 2006, p. 706)

A pesar de las amplias potencialidades de la educación continua, su contribución ha sido insuficientemente abordada en cuanto a su influencia en la formación integral del estudiantado de ingeniería. En esta investigación se postula que la educación continua posee muchas potencialidades que trascienden la simple actualización del profesional, ya que coexiste en las facultades de ingeniería con el proceso formativo estudiantil, por lo que ha de constituirse en una vía importante para complementar dicha formación en su tiempo de estudios. Por tanto, se puede afirmar que es un proceso formativo en sí mismo, pues posee objetivos concretos así como métodos y estrategias para su consecución.

\section{En torno a la formación integral del estudiantado de ingeniería}

La formación del estudiantado es un tema siempre actual. Su enfoque integral continúa en el centro de la atención de todas aquellas personas interesadas en contribuir, de manera significativa, al desarrollo socio-económico. Es reconocido que uno de los cimientos principales de un país es su sistema educativo, de aquí la preocupación por su modernización. En este sentido, la Organización para la Cooperación y el Desarrollo Económicos (OCDE), citada en (Fraile, 2007), expresa:

Los rápidos cambios económicos y tecnológicos de la sociedad actual, hace [sic] inútil una formación orientada exclusivamente hacia la formación profesional, pues la evolución de los conocimientos y la propia transformación de las empresas deja obsoletos sus contenidos. Además, la diversidad y heterogeneidad pluricultural de las sociedades postindustriales, exigen que los sistemas educativos permitan que el alumnado adquiera una serie de saberes y competencias para adaptarse al cambio. (p. 21)

Sin lugar a dudas, como refiere Cabrera (2013), "se nos presentan [sic] una situación compleja que nos invita a considerar nuestro contexto educativo [universitario] como un desafío 
no sólo profesional, sino también personal, en la que se requiere un pensamiento reflexivo y maduro que nos conecte mejor con nuestra conciencia" (p. 16). León y Ramírez (2010) indican que "las empresas buscarán y promoverán la formación de ingenieros con capacidades y entrenamientos especiales y variados para maximizar su potencial de éxito en las diversas culturas y situaciones aumentando la demanda de ingenieros que cuenten con características adicionales a sus habilidades técnicas" (p. 7).

Por las razones apuntadas, es comprensible que "un buen ingeniero o ingeniera" no es aquella persona que posee un alto dominio de la técnica, sino quien, partir de una profunda preparación en su especialidad, posee la sensibilización y compromiso de contribuir para mejorar la calidad de vida de sus contemporáneos, para lo cual precisa una formación más integral. Por ello, la formación integral se corresponde con el desarrollo equilibrado de la dimensión personal y profesional del estudiantado, manifestada en una unidad armónica en correspondencia con las exigencias de la sociedad actual.

A pesar de que la formación integral es multidisciplinar, hay aspectos que se consideran esenciales en cuanto a la cultura que se pretende desarrollar con el modelo por competencias. En este sentido, la formación integral se debe centrar en que la capacidad de aprendizaje de un estudiante se manifiesta en su habilidad para construir conocimiento de forma activa, cuya utilización no se limite a la solución de los problemas profesionales.

Además de demandar un carácter sistemático, la formación integral analizada se ha de comprender como un proceso consciente y de carácter continuo que transita gradualmente hacia lo interno del estudiante, hacia la adquisición de experiencias en términos de valores y de autodirección; por ello, constituye premisa y resultado del crecimiento de la personalidad en lo personal, lo profesional y lo social.

\section{Resultados de la etapa exploratoria acerca de las potenciales de la educación continua para formación integral del estudiante de ingeniería}

Para la realización de esta etapa se determinaron una serie de indicadores los cuales denotan la influencia que puede ejercer la educación continua en la formación integral del estudiantado de ingeniería. Estos indicadores incluyen aspectos relacionados con el conocimiento del significado y funciones de la educación continua por parte de docentes y estudiantes, la participación de ambos en las actividades, el nivel de satisfacción, contribución al desarrollo integral, así como la autoestima en la dimensión personal y el desarrollo de habilidades para el trabajo en equipo, en la dimensión social. 


\section{Conocimiento y participación en la educación continua (estudiantes)}

Para que la educación continua cumpla con sus funciones potenciales se requiere, ante todo, que el estudiantado posea conocimiento de su existencia y alcance. En la figura 1, se observa que el número de estudiantes que está informado sobre la existencia de la coordinación de educación continua y sus servicios es $60 \%$, mientras que el $40 \%$ reconoce tener poco conocimiento de dicha coordinación.

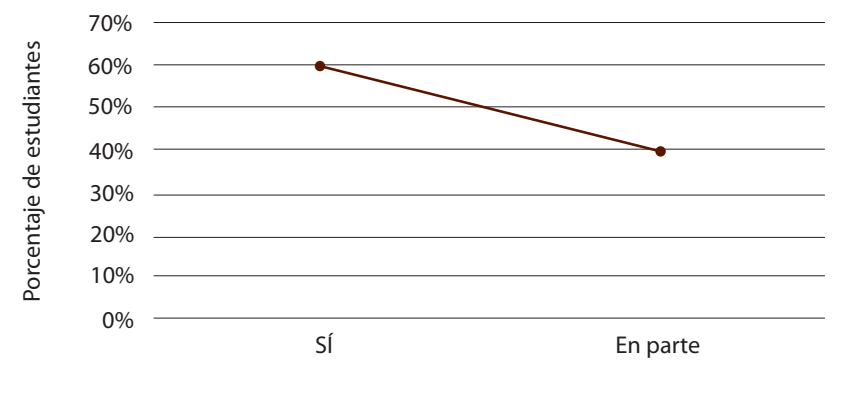

Conocimientos

Figura 1. Conocimiento sobre la existencia de la educación continua. Información derivada de las encuestas.

Por otra parte, fue de interés conocer la participación estudiantil en algún programa de la educación continua. Según muestra la figura 2, una minoría (34\%) ha tomado algún curso impartido por educación continua y un $66 \%$ no ha tomado ningún curso. Asimismo, en caso de responder afirmativamente, se especifica el curso. Este hecho indica que no se explotan estas posibilidades por partes de los estudiantes.

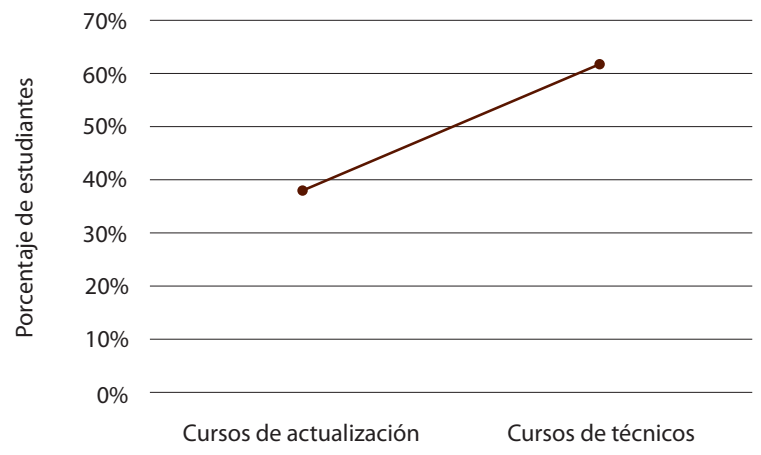

Tipo de cursos

Figura 2. Participación en los programas de educación continua. Información derivada de las encuestas. 
URL: http://www.una.ac.cr/educare

CORREO: educare@una.cr

Por otra parte, los resultados obtenidos muestran que el $75 \%$ de los participantes opina que es buena la participación de los profesores, mientras que el $15 \%$ contestó que la participación es regular, el 10\% refirió que la participación es mala (figura 3).

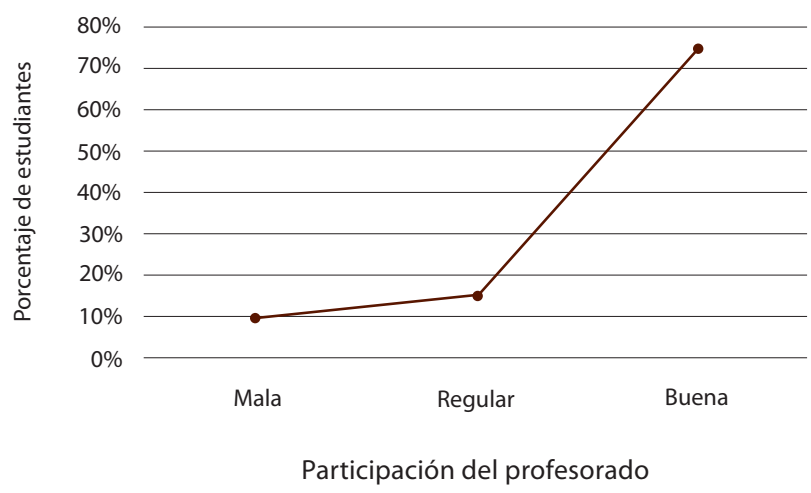

Figura 3. Participación del profesorado (según estudiantes). Información derivada de las encuestas.

\section{Aspectos de la dimensión social: trabajo en equipo}

Uno de los factores más preocupantes es el desarrollo social. Por ello, en las encuestas fue incluido este aspecto, para el cual se obtuvo como resultado que el $90 \%$ corrobora que existe una gran contribución al desarrollo social; el 10\% opina que existe, pero medianamente (figura 4).

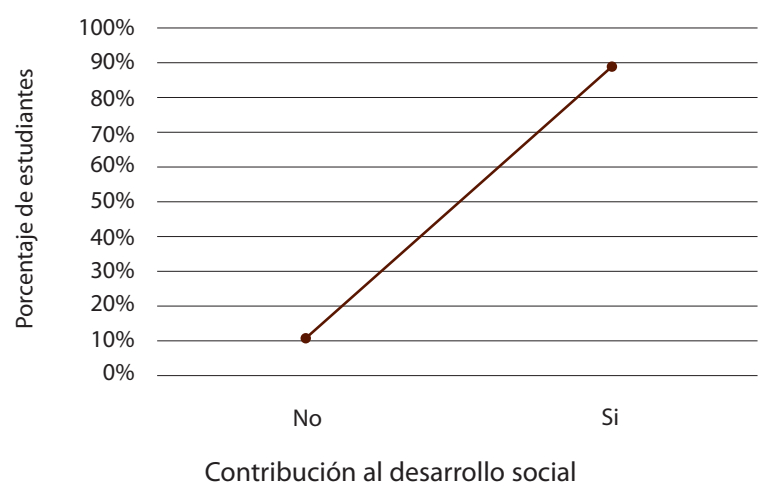

Figura 4. Contribución al desarrollo social. Información derivada de las encuestas. 
La figura 5 muestra la influencia de los programas de educación continua para facilitar el trabajo en equipo. En este caso, el $58 \%$ de los participantes afirma que participar en la coordinación fomenta el trabajo en equipo, el 39\% reconoció que de cierta manera influye al desarrollo del trabajo en equipo, mientras que solo el $3 \%$ opina que no influye en lo absoluto en la fomentación del trabajo equipo.

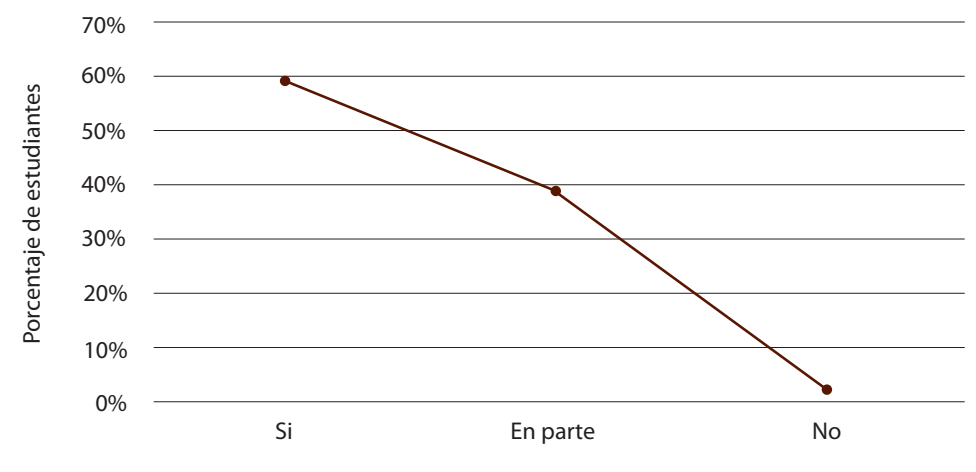

Fomento del trabajo en equipo

Figura 5. Criterios de los participantes sobre el fomento del trabajo en equipo en las actividades de educación continua. Información derivada de las encuestas.

\section{Aspectos de la dimensión personal: autoestima}

Como muestra la figura 6, la educación continua influye en la mejora de la autoestima de sus participantes. El 56\% expresa que influye, el 38\% afirma que solo algunas veces, el $6 \%$ opina que no influye en la mejora de la autoestima.

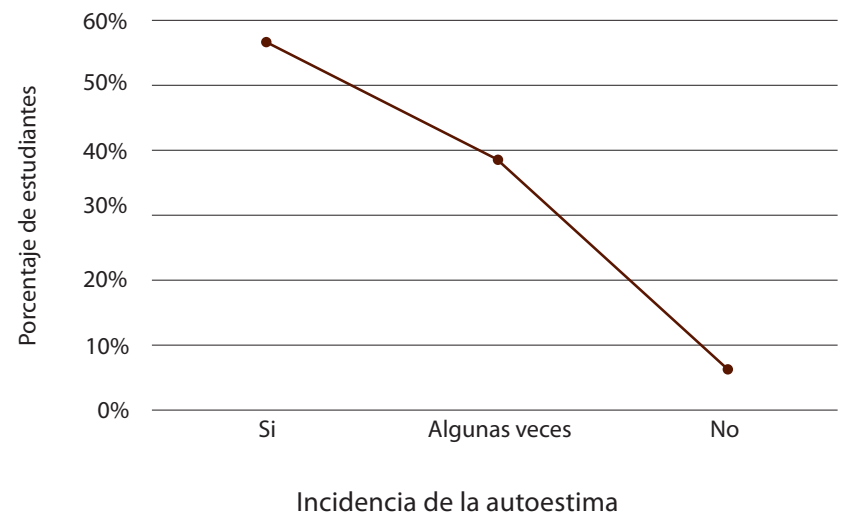

Figura 6. Evaluación sobre la mejora de la autoestima de los participantes de educación continua. Información derivada de las encuestas. 
URL: http://www.una.ac.cr/educare

CORREO: educare@una.cr

\section{Nivel de satisfacción del estudiantado respecto a la educación continua}

La figura 7 evidencia cómo fue autoevaluado el nivel de satisfacción de los participantes en programas de la educación continua. El 53\% opinó que los programas ofertados tienen un alto nivel de calidad, por otra parte, el $38 \%$ lo califica como medio, mientras que solo el $9 \%$ apunta que la calidad de los programas ofertados por educación continua es baja.

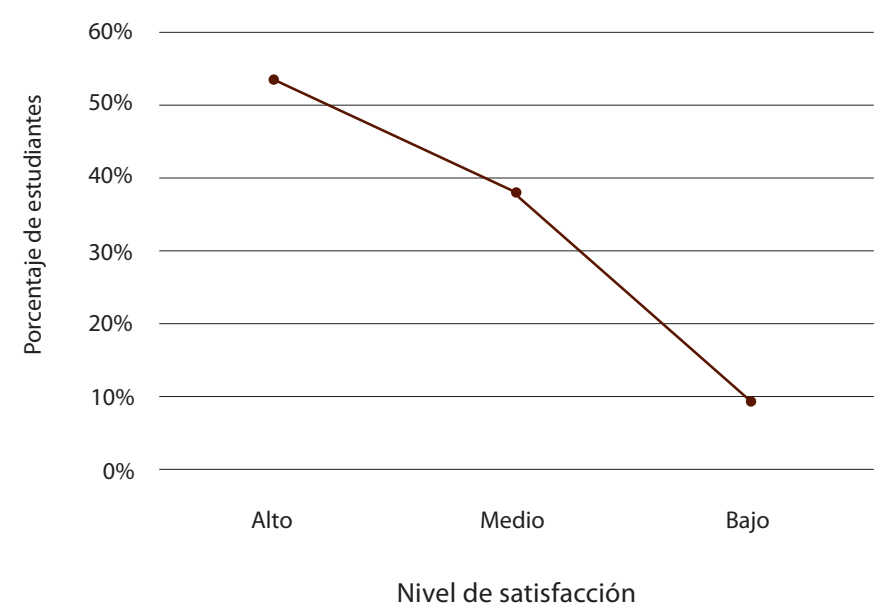

Figura 7. Nivel de satisfacción para los participantes de educación continua. Información derivada de las encuestas.

\section{Contribución de la educación continua al desarrollo integral}

En la figura 8 se observa que el $90 \%$ de participantes opina que la universidad promueve actividades de desarrollo integral, mientras un $10 \%$ contesta que a veces y ninguno de los encuestados desconoce estas actividades.

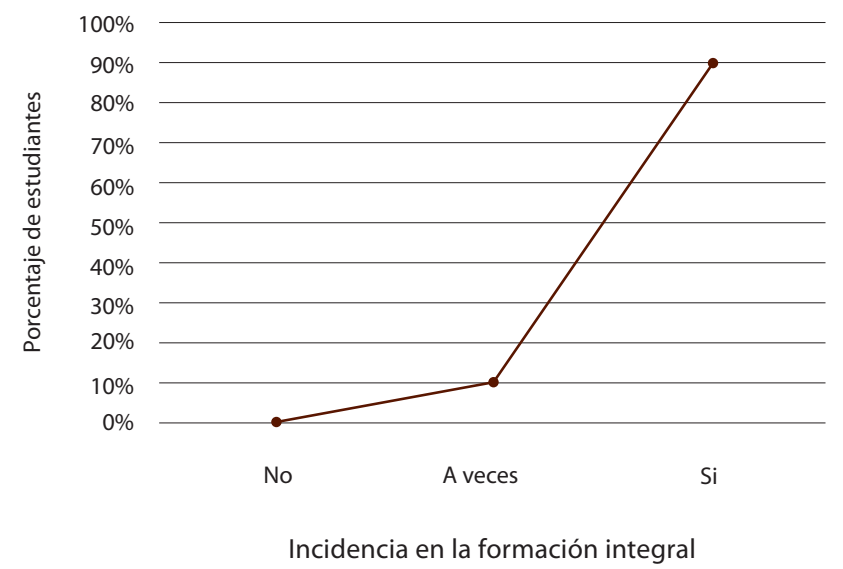

Figura 8. Contribución de la educación continua al desarrollo integral. Información derivada de las encuestas. 
Los estudiantes que contestaron afirmativamente mencionaron ejemplos entre los cuales destacan: prácticas profesionales, actividades con los becarios, las actividades de música como FIMECANTA, actividades altruistas, intercambios educativos, eventos externos, conciertos, actividades deportivas, diplomados y seminarios, grupos de deportes, actividades recreativas, informativas, talleres, eventos de las relaciones culturales, siembra de árboles, entre otras.

Respecto al desarrollo profesional y al desarrollo de relaciones a largo plazo se observa que el $85 \%$ de participantes plantea que la educación continua les ayuda mucho al desarrollo profesional, el 15\% manifiesta que medianamente. El $90 \%$ de los participantes establece muchas relaciones a largo plazo, y el 10\% las establece medianamente. El 95\% de los participantes declara que les ayuda mucho a formarse como mejores profesionales mientras el $5 \%$ opina que les ayuda medianamente. Al $85 \%$ de los participantes les ayuda mucho a estrechar lazos con el sector externo, mientras que al $15 \%$ les ayuda medianamente.

\section{Resultados de encuesta al profesorado}

\section{Conocimiento y participación en la educación continua}

En cuanto al conocimiento sobre la educación continua por parte del profesorado, la figura 9 muestra que el número de docentes que conocen las actividades orientadas a la formación integral dentro de la universidad es más del $87 \%$, mientras el $13 \%$ refiere que solo a veces se promueven estas actividades. De los encuestados que contestaron afirmativamente y proporcionaron ejemplos en el sentido apuntado, las más significativas fueron: servicio social (63\%) y servicio comunitario (37\%).

Las actividades integrales de mayor conocimiento por el profesorado son los intercambios académicos, mientras que los menos reconocidos fueron los temas curriculares.

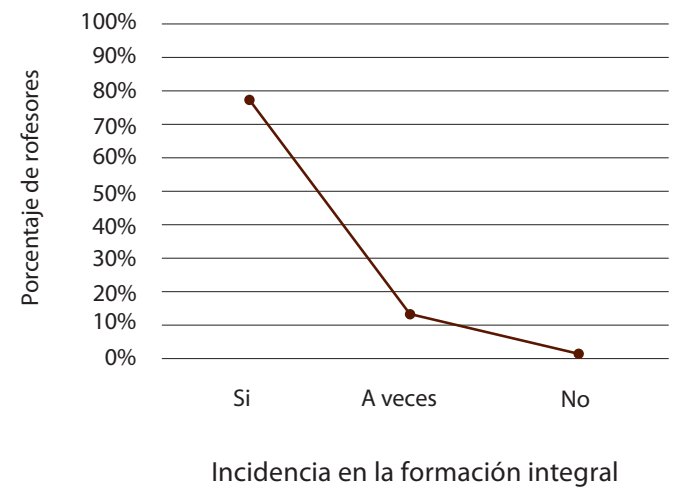

Figura 9. Criterios sobre la influencia de la educación continua en el desarrollo integral. Información derivada de las encuestas. 
URL: http://www.una.ac.cr/educare

CORREO: educare@una.cr

La tendencia de la participación de los profesores en actividades de desarrollo integral dentro de educación continua es muy baja, hecho que coincide con el criterio estudiantil. En su mayoría no se vinculan o es muy poca su participación. Solo el $30 \%$ de los profesores muestra una buena participación (figura 10).

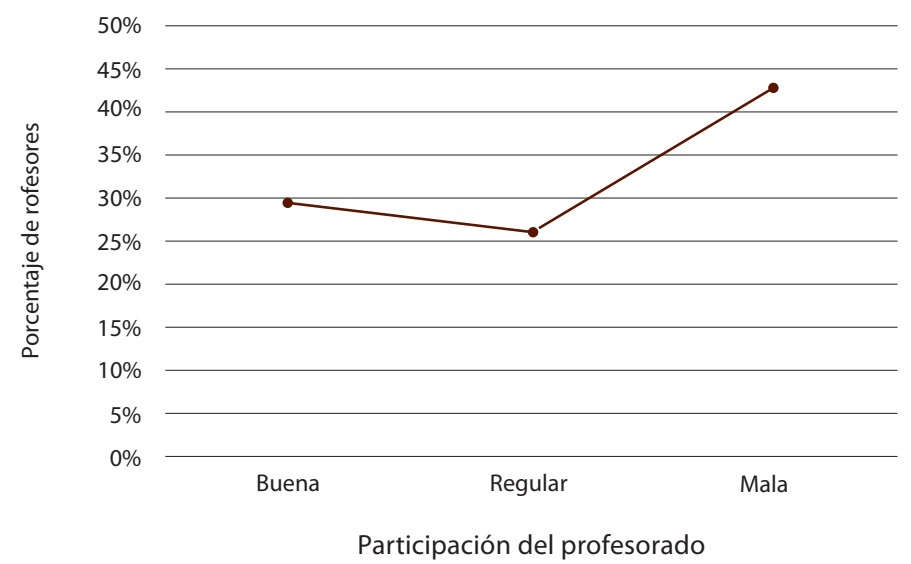

Figura 10. Participación de profesores en educación continua. Información derivada de las encuestas.

\section{Contribución de la educación continua al desarrollo integral}

Un 57\% de los profesores opina que las actividades de desarrollo integral dentro de la FIME son buenas, ya que este tipo de prácticas ha contribuido al desarrollo integral de participantes en educación continua. El $23 \%$ las valora de regular y el $20 \%$ no tienen conocimientos de las actividades ofertadas (figura 11).

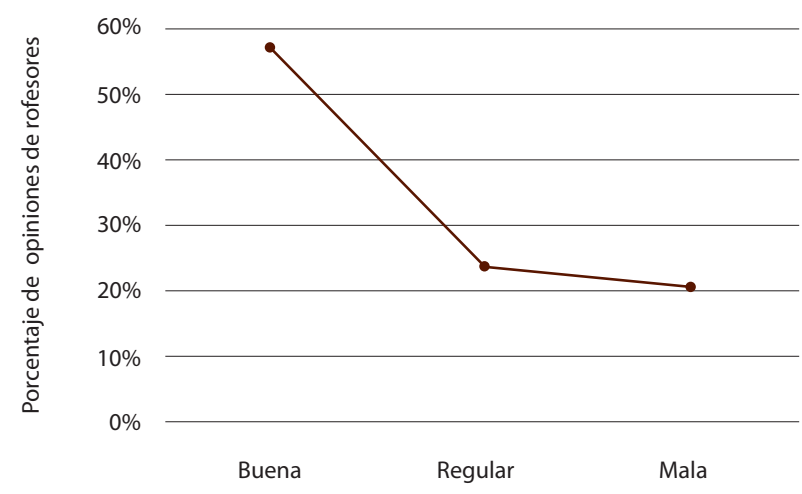

Incidencia de las actividades en el desarrollo integral

Figura 11. Actividades de desarrollo integral. Información derivada de las encuestas. 
La figura 12 refleja que un $46 \%$ del profesorado conoce los objetivos y funciones de educación continua y el desconocimiento es menos del 17\%. Sin embargo, se pretende que en un futuro la educación continua sea conocida por todos a través de una adecuada difusión. Los programas de educación continua operan con temas en un amplio espectro en la modalidad de cursos abiertos. Los docentes podrían desarrollar un alto grado de sistematización y vínculo con sectores productivos, así como en cuanto al conocimiento y relación con una empresa, cuestiones que favorecen el desarrollo profesional del estudiante aún en su tiempo de estudios.

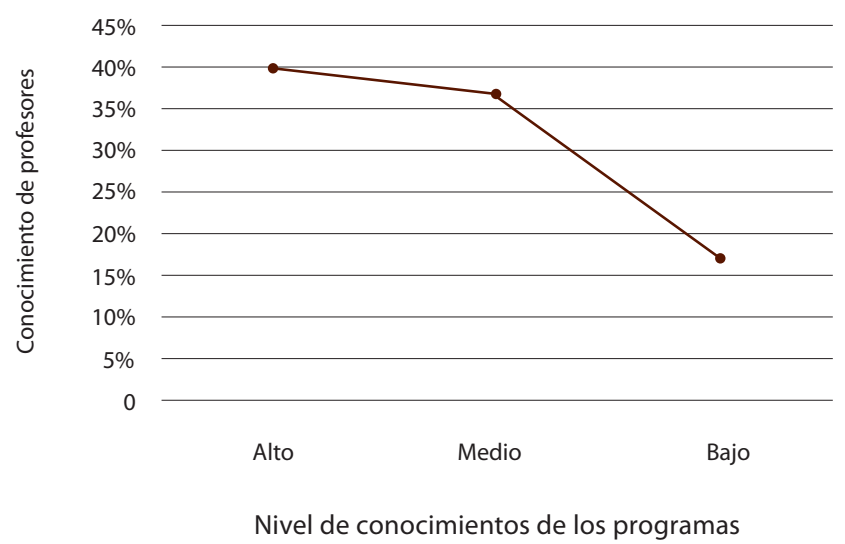

Figura 12. Programas de educación continua. Información derivada de las encuestas.

La tabla 1 muestra lo señalado por los profesores en cuanto a los principales aportes de la coordinación. Se puede observar que la percepción acerca de la educación continua es muy general y limitada.

Tabla 1

Variantes a valorar por el profesorado en cuanto al aporte de la educación continua

\begin{tabular}{lccc}
\hline Variantes/aportes de la educación continua & Mucho & Mediante & Nada \\
\hline Al desarrollo profesional & 17 & 4 & 0 \\
Establecer relaciones a largo plazo & 18 & 3 & 0 \\
A formar mejores profesionistas & 20 & 1 & 0 \\
A estrechar lazos con el sector externo & 17 & 2 & 2 \\
\hline
\end{tabular}


URL: http://www.una.ac.cr/educare

CORREO: educare@una.cr

\section{Aspectos de la dimensión social: trabajo en equipo}

Según los datos mostrados en la figura 13 (sobre el fomento del trabajo en equipo), en su mayoría opinan que permite adquirir esta habilidad (el $48 \%$ ), un $23 \%$ reconoce que a veces y un $29 \%$ afirmó no tener conocimiento.

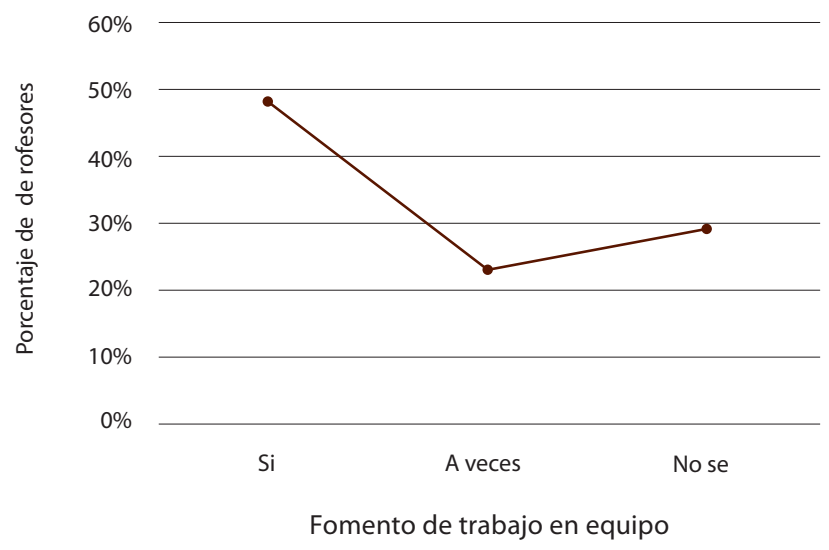

Figura 13. Fomento del trabajo en equipo en los participantes de educación continua. Información derivada de las encuestas.

\section{Conclusiones}

En la práctica educativa de las facultades de ingeniería, la educaciòn continua representa un proceso de formaciòn que se desarrolla de manera paralela al proceso formativo curricular, cuyas funciones se encaminan a concretar la formaciòn permanente de egresados de ingenería. A su vez, permite la preparación del estudiantado en múltiples aspectos en su tiempo de estudios. Sin embargo, la realidad muestra que no se aprovechan sus amplias posibilidades para la formaciòn integral. Este hecho es un elemento cardinal por tener en cuenta cuando se elaboren propuestas con este fin.

La etapa exploratoria de este estudio evidencia que existen dificultades para aprovechar las posibilidades que brinda esta área, debido a una concepciòn restringida y parcial acerca de esta, tanto por parte de profesores como de estudiantes. Estas limitaciones se observan aún cuando en las facultades de ingenierìa la educación continua se organiza en departamentos o coordinaciones, lo que facilita el acceso de todos los estudiantes en formación. Se observan insuficiencias que provocan una subutilización de esta vía para el logro de una formación trascendete de los futuros ingenieros e ingenieras. Es insuficiente la divulgaciòn de sus objetivos y posibilidades, ya que un porciento considerable de estudiantes y docentes los desconocen, lo que limita su influencia formativa y desarrolladora. 
El estudiantado que ha participado en actividades de la educación continua reconoce sus potencialidades para el desarrollo personal y profesional, dimensiones fundamentales de la formación integral de profesionales en ingeniería. Estos resulatdos contrastan con el significado tradicional atribuido a esta modalidad formativa.

Por estas razones se puede afirmar que una mayor incorporación del estudiantado, tanto en los cursos técnicos como en otras actividades en este tipo de coordinación, beneficiaría los resultados formativos en estas facultades.

\section{Referencias}

Álvarez, J. (mayo-agosto, 2002). La educación en valores. Emergencia del proceso formativo. Humanidades Médicas, 2(2), 1-33. Recuperado de http://scielo.sld.cu/scielo.php?pid=S1727$\underline{81202002000200005 \& \text { script }=\text { sci arttext }}$

Álvarez, N. (marzo, 2013). La renovación personal del docente: Premisa para un cambio radical en Pedagogía. Apuntes de Pedagogía, 4, 18-19. Recuperado de www.cdlmadrid.org/cdl/ htdocs/boletines/.../apuntespedagogia11.pdf

Asociación Nacional de Facultades y Escuelas de Ingeniería (ANFEI). (junio, 2000). La formación humanística del ingeniero. Conclusiones. Trabajo presentando en la XXVII Conferencia Nacional de Ingeniería. Instituto tecnológico de Toluca. Recuperado de http://www.anfei. org.mx/XXVII CNI/conclusiones XXVII CNI.pdf

Asociación Nacional de Universidades e Instituciones de Educación Superior (ANUIES). (2000). La educación superior en el siglo XXI. Líneas estratégicas de desarrollo. México: Autor. Recuperado de http://cedoc.infd.edu.ar/upload/La educacion superior en el siglo XXI.pdf

Cabrera J. (marzo, 2013). ¿Hacia dónde vamos? Reflexiones en torno a la renovación pedagógica. Apuntes de Pedagogía, 4, 16-17. Recuperado de: www.cdlmadrid.org/cdl/htdocs/boletines/.../ apuntespedagogia11.pdf

Consejo Federal de Decanos de Ingeniería (CONFEDI). (2006). Competencias genéricas. Desarrollo de competencias en la enseñanza de la ingeniería argentina (Primer acuerdo). San Juan: Facultad de Ingeniería, Universidad Nacional de San Juan.

Cornejo, J., Santilli H. B., Roble, M. B., Martín, A. M., Barrero, C. y Barbiric, D. (agosto, 2013). Las cuestiones éticas y sociales en la formación del ingeniero. Tres estudios de caso en bioingeniería. Revista Argentina de Enseñanza de la Ingeniería, 2(4), 7-15. Recuperado de http://www.ing.unrc.edu.ar/raei/archivos/img/arc 2013-09-02 $07 \quad 58$ 23-n4t01.pdf

De la Herrán, A. y Muñoz J. (2002). Educación para la universalidad. Más allá de la globalización. Madrid: Dilex. 
Fraile, A. A. (2007). La formación didáctica y profesional del profesorado de educación física. Revista Motricidad Humana, 4(1): 20-30. Recuperado de http://www.revistamotricidad.com/?rmh articulos=la-formacion-didactica-y-profesional-del-profesorado-de-educacion-fisica

León, A. y Ramírez, D. C. (junio, 2010). Visión prospectiva de la formación en ingeniería. Trabajo presentado en Eighth LACCEI Latin American and Caribbean Conference for Engineering and Technology (LACCEI'2010). "Innovation and Development for the Americas". Arequipa, Perú. Recuperado de http://www.laccei.org/LACCEl2010-Peru/published/Elnn023 Arenas. pdf

Mejía, A. (1986). Educación continua. Educación Médica y Salud, 20(1), 43-69. Recuperado de http://hist.library.paho.org/Spanish/EMS/6916.pdf

Méndez, D. (julio-diciembre, 2007). Nuevos retos en la formación de ingenieros. Revista Ciencias de la Educación, 17(30), 223-231. Recuperado de http://www2.scielo.org.ve/pdf/rce/v17n30/ art14.pdf

Pineda, P., y Sarramona, J. (setiembre-diciembre, 2006). El nuevo modelo de formación continua en España: Balance de un año de cambios. Revista de Educación, 341, 705-736. Recuperado de http://www.revistaeducacion.mec.es/re341/re341 29.pdf

Soto, L., Dimas, M. I. y Torres, A. (abril, 2012). Caracterización y perspectivas del proceso de formación del docente de la Facultad de Ingeniería Mecánica y Eléctrica de la UANL. Educación y futuro. Revista de investigación aplicada y experiencias educativas, 26, 241-260. Recuperado de http://dialnet.unirioja.es/servlet/ejemplar?codigo=303117

\section{Cómo citar este artículo en APA:}

Reynoso, M., Castillo, J. A. y Dimas, M. I. (enero-abril, 2014). La formación integral del estudiantado de ingeniería a través de la educación continua. Revista Electrónica Educare, 18(1), 77-96. Recuperado de http://www.revistas.una.ac.cr/index.php/EDUCARE/issue/current

Nota: Para citar este artículo en otros sistemas puede consultar el hipervínculo "Como citar el artículo" en la barra derecha de nuestro sitio web:

http://www.revistas.una.ac.cr/index.php/EDUCARE/index 